\title{
Tricuspid leaflet repair: innovative solutions
}

\author{
Jack H. Boyd ${ }^{1}$, J. James B. Edelman ${ }^{2}$, David H. Scoville ${ }^{1}$, Y. Joseph Woo ${ }^{1}$ \\ ${ }^{1}$ Department of Cardiothoracic Surgery, Stanford University School of Medicine, Stanford, CA, USA; ${ }^{2}$ Department of Cardiothoracic Surgery, \\ University of Sydney, Royal Prince Alfred Hospital, The Baird Institute, Sydney, Australia \\ Correspondence to: Jack H. Boyd, MD. Department of Cardiothoracic Surgery, Stanford University School of Medicine, Stanford, CA, USA. \\ Email: jackboyd@stanford.edu.
}

\begin{abstract}
Tricuspid regurgitation (TR) represents a significant disease process and when severe, is associated with increased mortality. Recent guidelines support a more aggressive approach to tricuspid valve (TV) surgery, especially when encountered with left-sided valvular pathology. While annuloplasty has been the standard treatment for TR, it may not provide as effective or durable a repair compared to annuloplasty combined with TV repair techniques. Several of these approaches are discussed including bicuspidalization, anterior leaflet augmentation, edge to edge repair, neochords, leaflet resection and combined approaches. Although patient cohorts in most of the studies examining these techniques are small, the long-term durability of TV repair is significant.
\end{abstract}

Keywords: Tricuspid valve repair; tricuspid leaflet repair techniques; tricuspid valve augmentation

Submitted Mar 24, 2017. Accepted for publication Mar 29, 2017.

doi: 10.21037 /acs.2017.05.06

View this article at: http://dx.doi.org/10.21037/acs.2017.05.06

\section{Introduction}

The decision to mechanically correct a valvulopathy remains a delicate balance between the risks and benefits. As the safety and efficacy of surgical and percutaneous approaches to the treatment of valvular diseases continue to improve, the threshold for intervention should lower. Following this rationale, increasing attention has been focused on the benefits of early treatment of tricuspid valve (TV) disease, especially when left-sided valves are being repaired. Increasing tricuspid regurgitation (TR) severity is associated with worse survival, independent of ventricular function, pulmonary artery pressures and age. As recently as a decade ago, 4-year survival in patients with severe TR was $64 \%$, compared with $>90 \%$ survival in patients with mild or less TR (1). Primary disorders of the TV include rheumatic heart disease, degenerative, traumatic, infective endocarditis, carcinoid syndromes and those associated with implanted pacemaker or defibrillator leads. The majority $(80 \%)$ of TV disorders, however, are functional and due to annular dilatation or leaflet tethering, resulting from volume or pressure overload. Furthermore, progression of TR after correction of left-sided valvular lesions is common (2).
The AHA/ACC have recently updated guidelines for TV surgery (3) with a trend toward a more aggressive approach. TV surgery is indicated in patients with severe TR undergoing left-sided heart surgery (class I); mild or moderate TR with annular dilatation and heart failure undergoing left-sided valve surgery (class IIa); or moderate TR with elevated PASP at the time of left-sided heart surgery (class IIb). Similarly, TV surgery can be considered in patients with severe primary TR unresponsive to medical therapy (class IIa) and considered in asymptomatic patients with severe primary TR with evidence of right ventricular dilatation or systolic dysfunction (class IIb).

\section{Repair versus replace}

Recognizing that the patient populations undergoing TV repair and TV replacement are different, peri-operative and long-term outcomes for TV replacement are inferior to those of repair. The peri-operative mortality after TV replacement has been reported to be as high as $20 \%$ in high-risk patients, those receiving re-operations after left sided surgery or those with severe symptoms and unrepairable valves. Risk factors identified for early death 
include age and left-sided valvular disease. This supports the notion that functional TR requiring replacement is a marker for end-stage valvular heart disease $(4,5)$. Indeed, the most common cause of peri-operative death following TV replacement is right ventricular failure (4).

In contrast, adding a TV procedure to left-sided heart surgery does not increase the risk of surgery. The use of TV annuloplasty rings rather than replacement for TV annular dilatation decreases the morbidity rates associated with leftsided prostheses- $3.83 \%$ per patient year for biological valves, $4.6 \%$ per patient year for mechanical valves (6).

\section{Indications for leaflet repair}

The use of ring alone is usually sufficient in most cases of TV repair, as the mechanism is commonly annular dilatation. Recurrence of TR, however, is not insignificant. In one series of patients ( $74 \%$ functional TR) treated with annuloplasty ring alone (performed as a concomitant procedure) the rate of moderate to severe TR at 5.9-year follow-up was $30 \%(7)$. Interestingly, approximately $1 / 5^{\text {th }}$ of patients $(22.6 \%)$ diagnosed with functional TR on preoperative echo are found to have organic or primary TV disease intraoperatively (7). Patients with primary disease of the tricuspid leaflets or subvalvular structures-those with ruptured chordae, trauma, endocarditis, or rheumatic heart disease-are unlikely to be adequately repaired with annuloplasty ring alone. Described below are techniques and results of TV repair procedures that can be used in conjunction with annuloplasty rings for more complicated repairs of diseased TVs with primary and mixed etiologies.

\section{Leaflet repair techniques}

A number of techniques have been described in case reports and small series but there is relatively little data to assess their efficacy. Several of the methods were initially developed for mitral valve repair.

\section{DeVega annuloplasty}

The DeVega repair technique was originally described in 1972 (8). It utilized a suture annuloplasty involving the annulus overlying the anterior and posterior leaflets. A running suture was initiated at the annulus near the septalposterior commissure and continued to the fibrous trigone. A pledget was then placed and the suture was reversed with the second layer intercalating with the first (Figure 1).

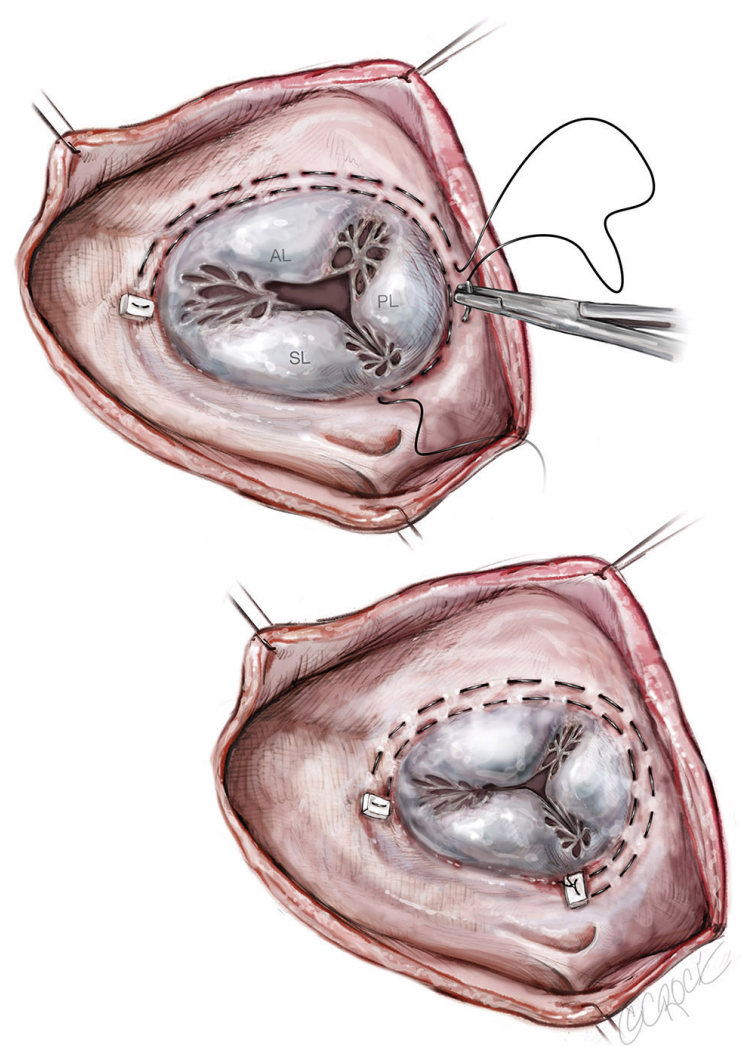

Figure 1 DeVega suture annuloplasty.

The suture was then tied over a sizing device. A number of studies have been performed and demonstrate a more durable repair with ring annuloplasty when compared with suture annuloplasty $(9,10)$.

\section{Bicuspidalization}

Originally described in 1965 by Kay et al., this technique employs a double pledget-supported mattress of a 2-0 braided suture that is placed along the annulus from the antero-posterior commissure to the postero-septal commissure (11). A $29-\mathrm{mm}$ valve sizer is placed in the valve, onto which the suture is tied down to obliterate the posterior leaflet (Figure 2). The sizer prevents there being too much strain on the annuloplasty repair (12). This posterior annuloplasty eliminates the unsupported area of the tricuspid annulus, where much of the dilatation occurs.

Ghanta and colleagues compared 237 patients who underwent either a bicuspidalization or ring annuloplasty of the TV as part of a concomitant procedure for treatment of functional TR with left-sided valve surgery (12). At 3 years, 


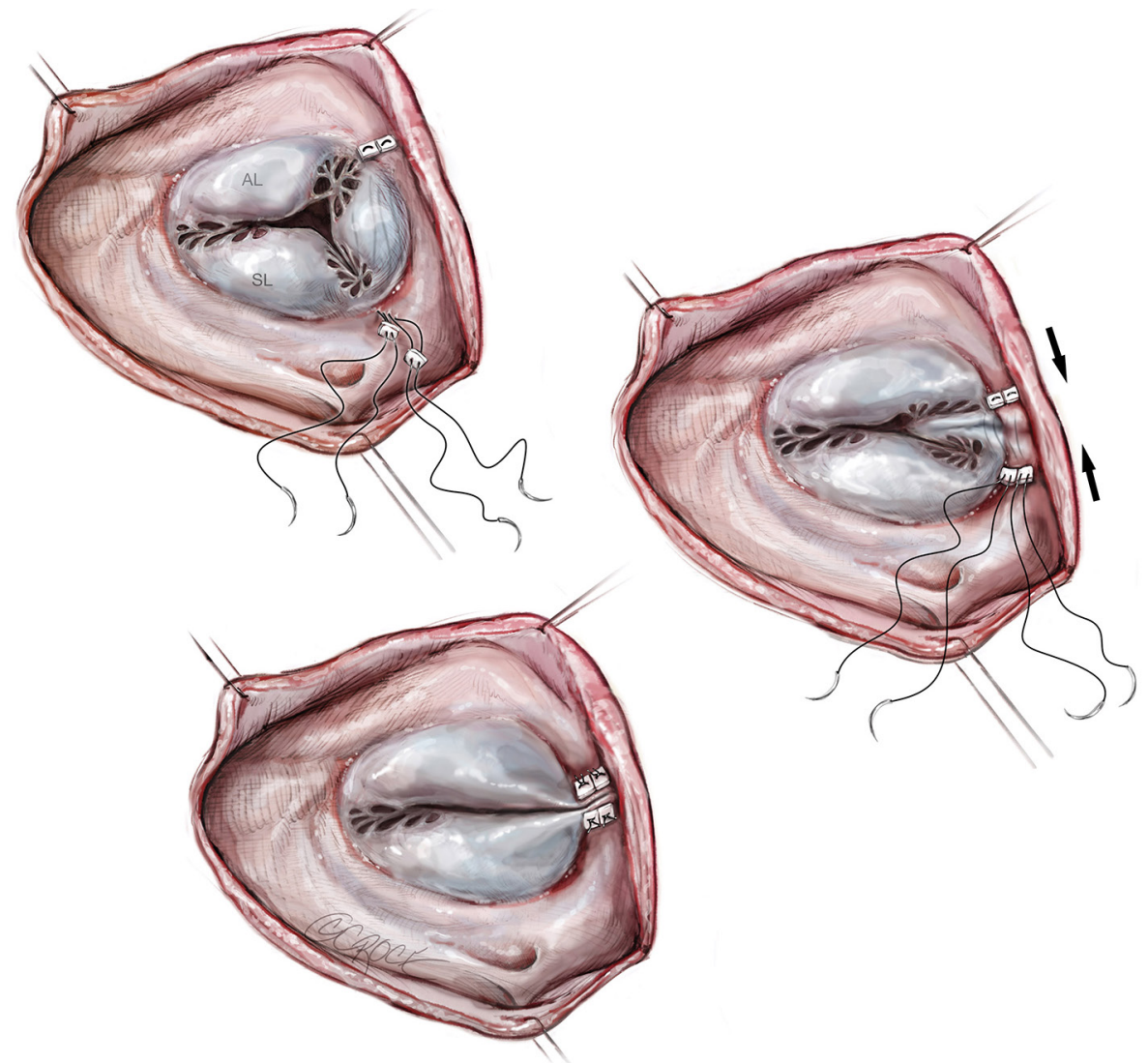

Figure 2 Bicuspidalization of TV by obliteration of the posterior leaflet and placement of annuloplasty ring.

TR was at least moderate in $25 \%$ of those who underwent bicuspidalization, compared with $31 \%$ of those with a ring annuloplasty $(\mathrm{P}=0.18)$. There was no difference in early or late survival or late functional status.

\section{Anterior leaflet augmentation}

Augmentation of the anterior leaflet can be used for leaflet tethering or retraction, as seen in severe functional TR (migration of the papillary muscles with RV dilatation) and rheumatic heart disease.

A curvilinear incision is made along the base of the anterior leaflet between antero-septal and antero-posterior commissures. The width and length of the deficit created is measured with the edge of the anterior leaflet, under enough traction to meet the septal annulus. A patch of autologous pericardium (bathed in glutaraldehyde for 10 minutes) is cut to measure the width $+0.5 \mathrm{~cm}$ and length $+0.5 \mathrm{~cm}$. The patch is then sewn into the anterior leaflet defect with 5-0 polypropylene suture. A saline pressure test is used to test elimination of the antero-septal malcoaptation. An annuloplasty ring is placed to improve coaptation and prevent annular dilatation (Figure 3) $(13,14)$.

Dreyfus and colleagues have reported results in 15 patients with severe functional TR and evidence of leaflet tethering undergoing left-sided procedures using this technique. There was no TR at the end of the procedure and a coaptation length of at least $5 \mathrm{~mm}$ was achieved in all patients (14).

Tang and colleagues have reported follow-up to 3.5 years in 31 patients with rheumatic TV disease and severe TR undergoing this procedure (with left-sided valve procedures). Discharge echocardiogram showed no greater than mild TR. At follow-up, there was moderate TR in 1 patient (3.2\%) but mild or less TR in the remaining patients (13).

\section{Edge-to-edge repair}

This approach, initially described by Alfieri and colleagues in the treatment of mitral regurgitation, has been adapted 


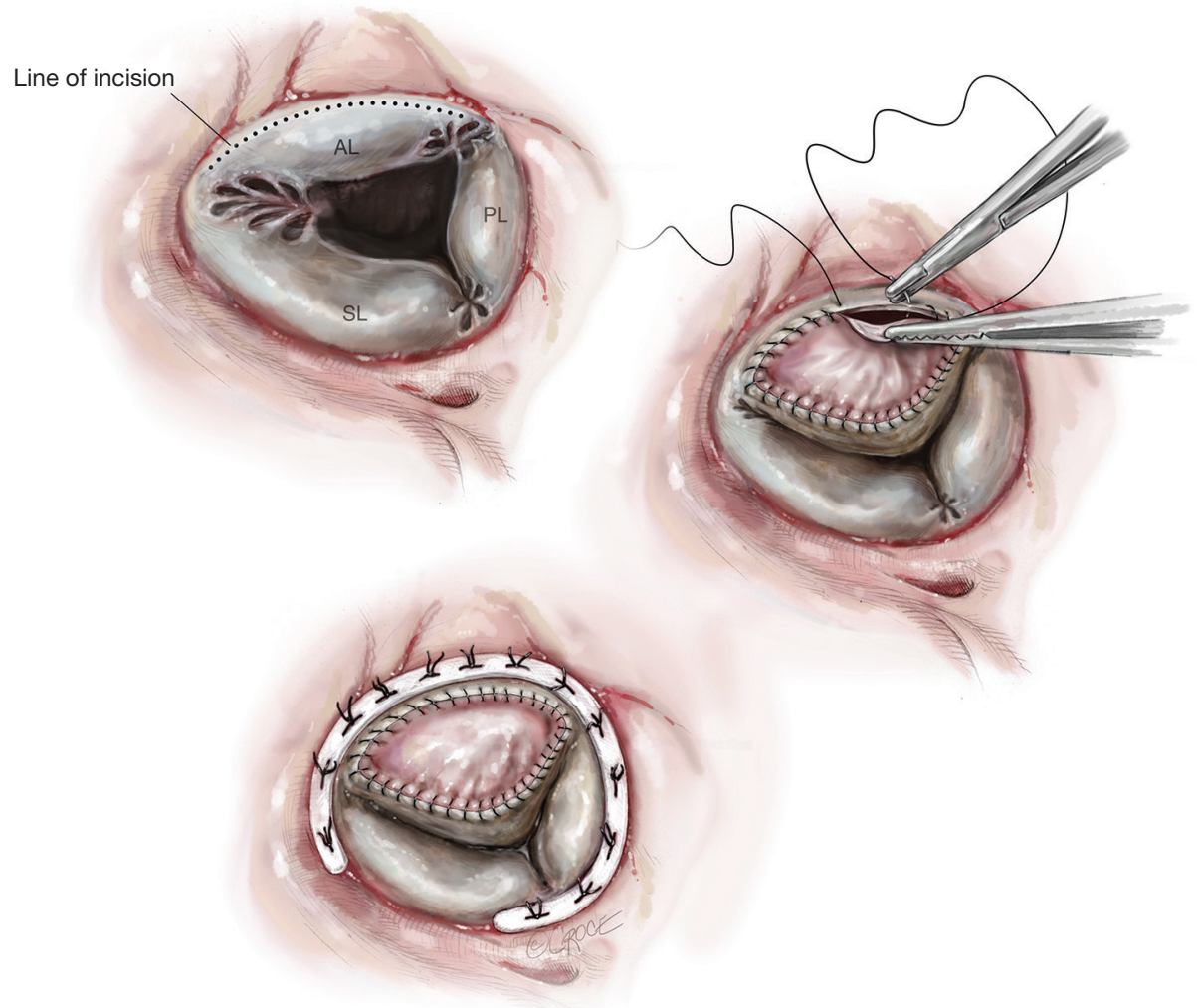

Figure 3 Anterior leaflet augmentation with annuloplasty ring.

for treatment of TR (15). It can be used to address residual regurgitation present after ring annuloplasty or other repair procedures (leaflet augmentation, leaflet transfer, chordal replacement). Alfieri originally described the technique for use in post-traumatic TR, with chordal or papillary muscle rupture.

The midpoints of the TV leaflets are stitched together, originally described without (15) but can be done with pledgets (5). This produces a triple orifice 'clover' repair, which was supplemented with an annuloplasty ring, if not performed already (Figure 4).

Lai and colleagues reported a series of 15 patients with severe TR of rheumatic, congenital, traumatic and degenerative origin. They utilized the edge-to-edge repair after the initial attempt at repair failed. The failed repairs consisted of annuloplasty in conjunction with other leaflet repair techniques. All cases had mild or less TR at the conclusion of the case. The mean transvalvular gradient ranged from 1.6 to $4.8 \mathrm{mmHg}$ (mean $3 \mathrm{mmHg}$ ), with a valve orifice area of $2.5-4 \mathrm{~cm}^{2}$. At 25.4 months follow-up, there was trivial-mild TR in 12 cases, and mild-moderate TR in two cases. One patient died peri-operatively (5).
Alfieri's group described the results of the technique in 14 patients with severe TR from a range of etiologies, specifically degenerative, post-traumatic and functional. On discharge, TR was no more than mild in all patients (absent in 46\%). One patient died peri-operatively. At 12 months follow-up, TR was mild or less in all but one patient (moderate). The mean valve area was $4.2 \mathrm{~cm}^{2}$, and the mean transvalvular gradient was $2.7 \mathrm{mmHg}$. Two patients had exercise stress echocardiography to assess the valve gradient; this remained below $3 \mathrm{mmHg}$ in both patients (16).

\section{Neochords}

There are case reports on the use of neochord repairs of the TV, but no long-term results are available. Marin and colleagues described a case using artificial polytetrafluoroethylene sutures to treat prolapse of the A1A2 portion of the TV anterior leaflet (17). An annuloplasty ring was placed in conjunction with neochords (Figure 5).

Chu and colleagues reported results of 12 patients undergoing neochord repair of the TV damaged by infective endocarditis (18). After debridement of the 


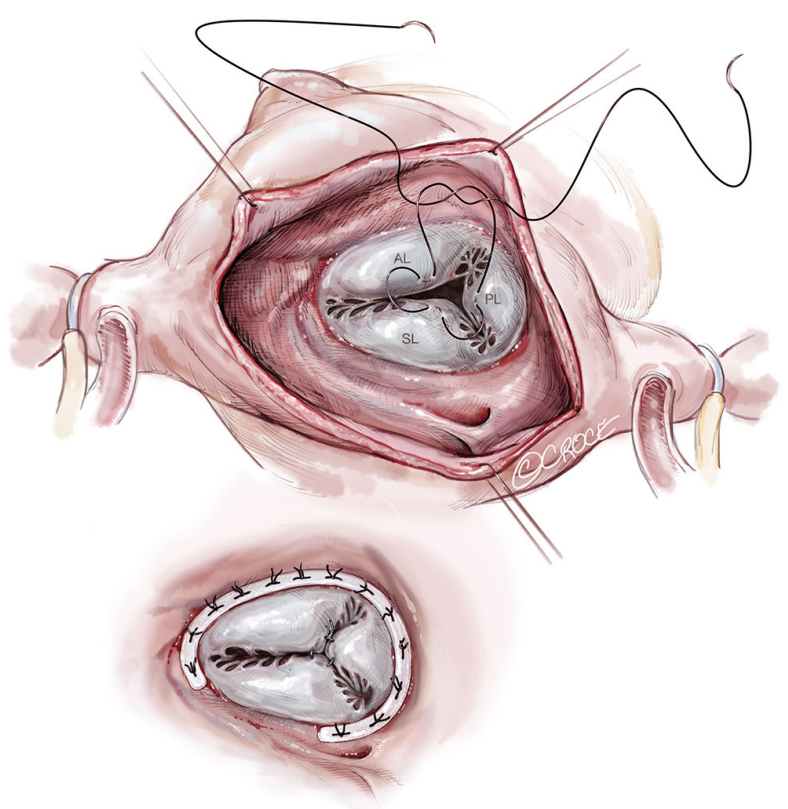

Figure 4 Edge to edge repair.

infected and damaged tissue, an autologous glutaraldehydetreated pericardial patch was prepared. Annuloplasty sutures were placed. The anterior and posterior leaflets were then reconstructed with one large patch, sewing the medial and lateral aspects to the anterior leaflet and the base to the annulus. The lateral aspect was attached to the residual posterior leaflet. Most of the coaptation occurs with the septal leaflet. Gore-Tex neochords were constructed from the papillary muscles to the free edge of the neo-leaflet. An annuloplasty ring was then placed to support the repair. There were no peri-operative deaths. All patients had mild or less TR at the conclusion of the case. At 3 months, two patients had moderate TR, but others $(83.3 \%)$ had no more than mild TR.

\section{Quadrangular resection}

Kim and colleagues reported the use of quadrangular resection to repair a TV damaged by infective endocarditis (19). The debridement of the anterior leaflet resembled a quadrangular resection of the posterior leaflet of a mitral valve. The remaining leaflet was approximated and sutured; the annulus was repaired with a De Vega annuloplasty (Figure 6). Substitution of an annular ring for the De Vega annuloplasty seems logical. TR was trivial on echocardiogram performed 8 years after operation.

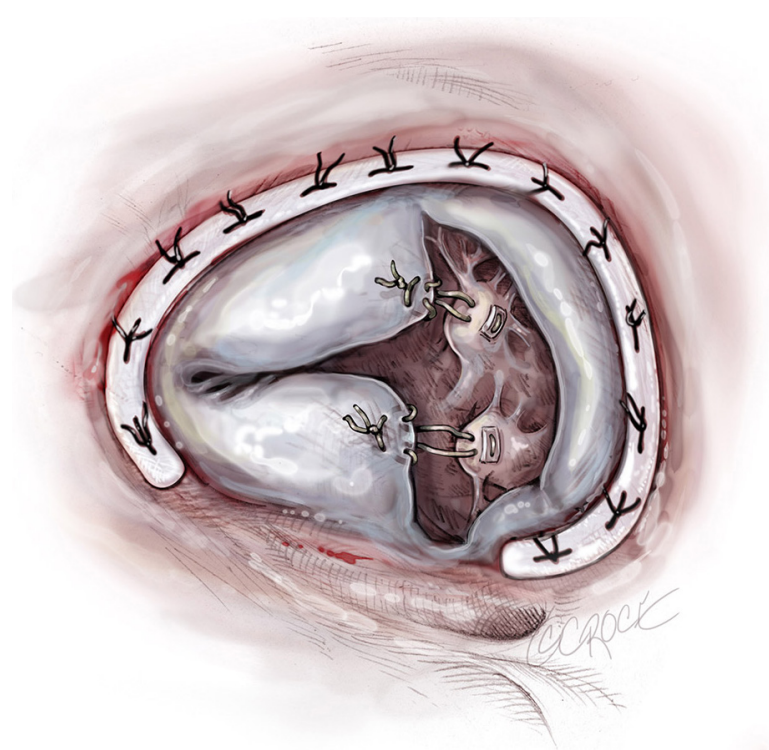

Figure 5 Placement of neochords.

\section{Multiple techniques}

$\mathrm{Ma}$ and colleagues reported results of 13 patients with traumatic TR undergoing valve repair using a combination of techniques: simple prolene repair of the lacerated leaflet, neochords, re-implantation of the papillary muscle to the $\mathrm{RV}$ free wall, edge-to-edge technique, leaflet plication (leaflet with ruptured chordae was sewn to adjacent normal leaflet or autologous pericardial patch repair/augmentation) and annuloplasty (20). There were no early or late deaths. At follow-up of mean 6.5 years, all patients were in NYHA I or II. Eleven patients had no more than mild TR, two had mild-moderate TR.

\section{Conclusions}

TR represents a significant disease process that when severe becomes a harbinger of mortality. With continuing improvements in outcomes, guidelines for valvular intervention will support early and more aggressive surgical treatment, including for those patients with TR especially in conjunction with left-sided valve repair. Although annuloplasty alone may improve functional TR, a better and longer lasting repair may be attained through combining annuloplasty with one of the aforementioned TV repair techniques. Furthermore, while the etiology of TR in most patients is functional, a substantial number have a primary cause and hence will not benefit from annuloplasty. Should repair be unsatisfactory with a ring alone, we suggest use of one or a combination of the techniques 

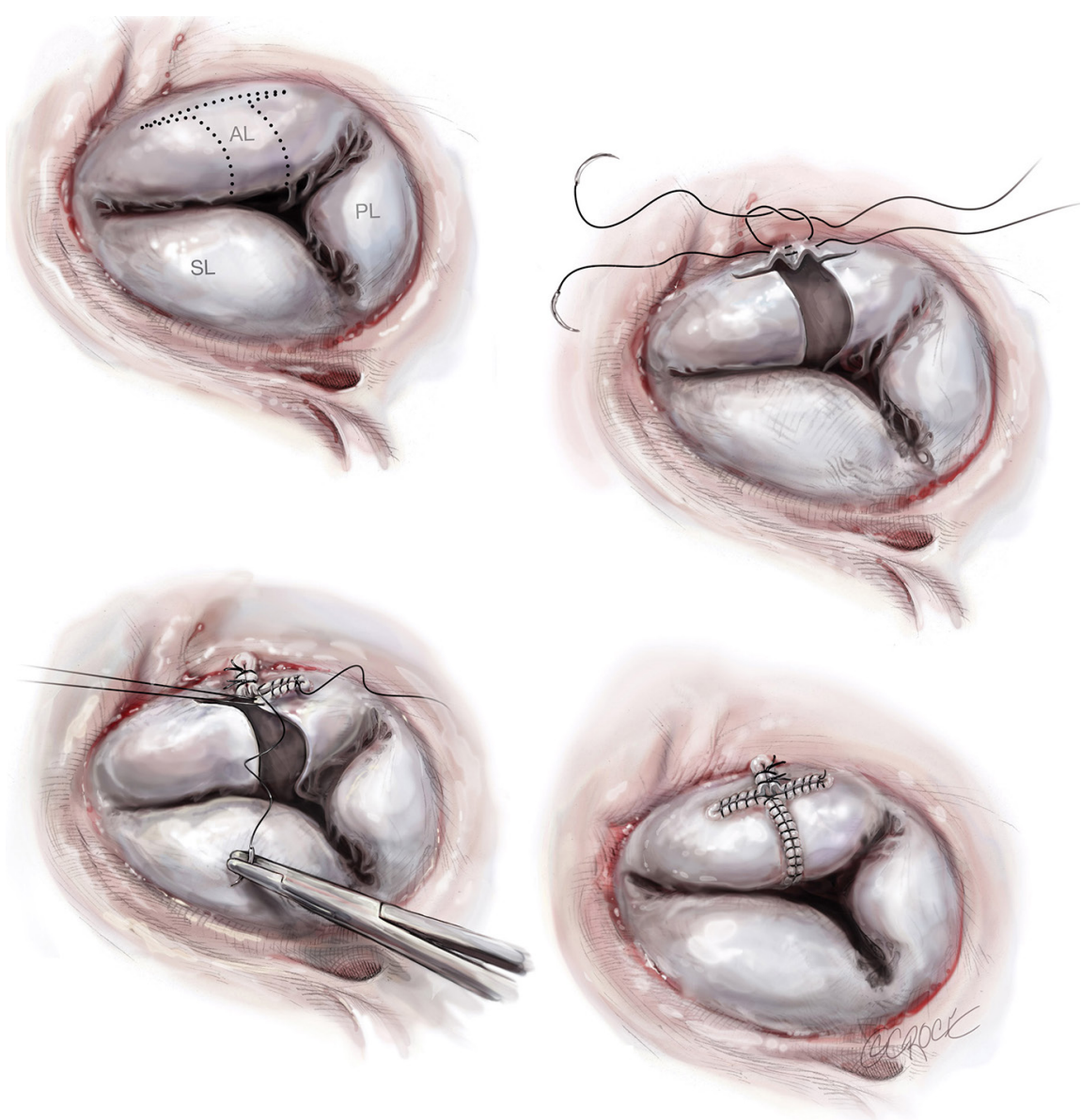

Figure 6 Quadrangular resection of the septal leaflet of the TV.

described above to supplement and improve the repair. Although patient cohorts in most of the studies are small, the long-term durability of TV repair techniques is significant. As TR repair increases, a more statistical comparison of these techniques will be possible.

\section{Acknowledgements}

None.

\section{Footnote}

Conflicts of Interest: The authors have no conflicts of interest to declare.

\section{References}

1. Nath J, Foster E, Heidenreich PA. Impact of tricuspid regurgitation on long-term survival. J Am Coll Cardiol 2004;43:405-9.

2. Goldstone AB, Howard JL, Cohen JE, et al. Natural history of coexistent tricuspid regurgitation in patients with degenerative mitral valve disease: implications for future guidelines. J Thorac Cardiovasc Surg 2014;148:2802-9.

3. Nishimura RA, Otto CM, Bonow RO, et al. 2014 AHA/ ACC guideline for the management of patients with valvular heart disease: a report of the American College of Cardiology/American Heart Association Task Force on Practice Guidelines. J Thorac Cardiovasc Surg 2014;148:e1-e132.

4. Filsoufi F, Anyanwu AC, Salzberg SP, et al. Long-term outcomes of tricuspid valve replacement in the current era. Ann Thorac Surg 2005;80:845-50.

5. Lai YQ, Meng X, Bai T, et al. Edge-to-edge tricuspid valve repair: an adjuvant technique for residual tricuspid regurgitation. Ann Thorac Surg 2006;81:2179-82. 
6. Chang BC, Lim SH, Yi G, et al. Long-Term Clinical Results of Tricuspid Valve Replacement. Ann Thorac Surg 2006;81:1317-23; discussion 1324

7. Tang GH, David TE, Singh SK, et al. Tricuspid valve repair with an annuloplasty ring results in improved longterm outcomes. Circulation 2006;114:I577-81.

8. DeVega NG. Selective, adjustable and permanent annuloplasty. An original technic for the treatment of tricuspid insufficiency. Rev Esp Cardiol 1972;25:555-6.

9. Huang X, Gu C, Men X, et al. Repair of functional tricuspid regurgitation: comparison between suture annuloplasty and rings annuloplasty. Ann Thorac Surg 2014;97:1286-92.

10. Parolari A, Barili F, Pilozzi A, et al. Ring or suture annuloplasty for tricuspid regurgitation? A meta-analysis review. Ann Thorac Surg 2014;98:2255-63.

11. Kay JH, Maselli-Campagna G, Tsuji KK. Surgical treatment of tricuspid insufficiency. Ann Surg 1965;162:53-8.

12. Ghanta RK, Chen R, Narayanasamy N, et al. Suture bicuspidization of the tricuspid valve versus ring annuloplasty for repair of functional tricuspid regurgitation: midterm results of 237 consecutive patients. J Thorac Cardiovasc Surg 2007;133:117-26.

13. Tang H, Xu Z, Zou L, et al. Valve repair with autologous pericardium for organic lesions in rheumatic tricuspid valve disease. Ann Thorac Surg 2009;87:726-30.

Cite this article as: Boyd JH, Edelman JJ, Scoville DH, Woo YJ. Tricuspid leaflet repair: innovative solutions. Ann Cardiothorac Surg 2017;6(3):248-254. doi: 10.21037/ acs.2017.05.06
14. Dreyfus GD, Raja SG, John Chan KM. Tricuspid leaflet augmentation to address severe tethering in functional tricuspid regurgitation. Eur J Cardiothorac Surg 2008;34:908-10.

15. Alfieri O, De Bonis M, Lapenna E, et al. The "clover technique" as a novel approach for correction of posttraumatic tricuspid regurgitation. J Thorac Cardiovasc Surg 2003;126:75-9.

16. De Bonis M, Lapenna E, La Canna G, et al. A novel technique for correction of severe tricuspid valve regurgitation due to complex lesions. Eur J Cardiothorac Surg 2004;25:760-5.

17. Marin D, Ramadan K, Hamilton C, et al. Tricuspid valve repair with artificial chordae in a 72-year-old woman. Thorac Cardiovasc Surg 2011;59:495-7.

18. Tarola CL, Losenno KL, Chu MW. Complex tricuspid valve repair for infective endocarditis: leaflet augmentation, chordae and annular reconstruction. Multimed Man Cardiothorac Surg 2015;2015:mmv006.

19. Kim JH, Kim YS, Yoon YH, et al. Quadrangular Resection of the Tricuspid Valve. Korean J Thorac Cardiovasc Surg 2013;46:60-2.

20. Ma WG, Luo GH, Sun HS, et al. Surgical treatment of traumatic tricuspid insufficiency: experience in 13 cases. Ann Thorac Surg 2010;90:1934-8. 